\title{
Efficacy of Yiqi Fumai (Freeze-Dried Powder) on Ischemic Diastolic Heart Failure
}

\author{
Dalin Song ${ }^{1,2}$, Mengfen $\mathrm{Hu}^{2}$, Tongliang Han1, Hua Zhang1, Yongjun Mao², Tao Tian ${ }^{3 *}$ \\ ${ }^{1}$ Department of Geriatric Cardiology, Geriatric Institute, Qingdao Municipal Hospital, Qingdao, China \\ ${ }^{2}$ Qingdao University, Qingdao, China \\ ${ }^{3}$ Department of Geriatric Cardiology, Linyi People's Hospital, Linyi, China \\ Email: dsong55cn@aliyun.com
}

Received 14 March 2014; revised 17 April 2014; accepted 6 May 2014

Copyright (C) 2014 by authors and Scientific Research Publishing Inc.

This work is licensed under the Creative Commons Attribution International License (CC BY). http://creativecommons.org/licenses/by/4.0/

(c) (;) Open Access

\begin{abstract}
Objective: To evaluate the efficacy of Yiqi Fumai freeze-dry powder (YFP) on ischemic diastolic heart failure. Methods: 100 patients diagnosed with unstable angina accompanying ischemic diastolic heart failure (IDHF) were selected randomly. 52 patients with TCM syndrome of qi-yin deficiency were divided into Chinese and Western combination therapy group. 48 patients have no TCM syndrome of qi-yin deficiency, and were treated with standard western medicine. After treatment, Seattle Angina Questionnaire Evaluation, ECG, conventional and stress echocardiography (SE) index, NT-proBNP were compared between before and after treatment. Results: The differences of PL, TS, DP between before and after treatment by YFP were statistically significant $(P<$ 0.05). SE parameters of LVEF, E/A were significantly increase; $E / e^{\prime}$ decreased. The differences were statistically significant. However, echocardiography parameters showed no significant differences after treatment. After the combination treatment, NT-proBNP level had negative correlation with LVEF, E/A ( $r=-0.432,-0.643$, both $P<0.01)$. Conclusion: Yiqi Fumai freeze-dry powder is safe and effective to patients with ischemic diastolic heart failure. Stress echocardiography can improve the diagnostic of ischemic diastolic heart failure, and maybe an effectively predict treatment response.
\end{abstract}

\section{Keywords}

Yiqi Fumai (Freeze-Dried Powder), Diastolic Heart Failure, Seattle Angina Questionnaire (SAQ) Evaluation, Stress Echocardiography

\section{Introduction}

Cardiovascular events are major fatal disease in China. Acute myocardial infarction (AMI) and ischemic heart

${ }^{*}$ Corresponding authors.

How to cite this paper: Song, D.L., Hu, M.F., Han, T.L., Zhang, H., Mao, Y.J. and Tian, T. (2014) Efficacy of Yiqi Fumai (Freeze-Dried Powder) on Ischemic Diastolic Heart Failure. Chinese Medicine, 5, 75-86.

http://dx.doi.org/10.4236/cm.2014.52009 
failure (IHF) are the most common and most serious stage in cardiac development. Unstable angina is one type of the acute coronary syndrome and its pathogenesis is unstable coronary plaque that plaque rupture may develop acute myocardial infarction. Myocardial ischemia and wide degeneration, necrosis and fibrosis caused by coronary artery disease can result in myocardial systolic and (or) diastolic dysfunction. Diastolic function occurs prior to systolic function. Diastolic heart failure (DHF) can be further developed to systolic heart failure, which has serious impact on cardiac function and quality of life of patients. Therefore, combination treatment of standardized western medicine joint proprietary Chinese medicine has been increasingly accepted by clinicians.

Coronary heart disease is divided into two categories, thoracic obstruction and angina pectoris, in traditional Chinese medicine (TCM). Both types of coronary disease are severe and require urgent intervention. The pathogenesis of coronary heart disease mainly involves heart vessel blockage or stasis. Delay in the treatment of this chronic disease may cause damage to Yin and Yang as well as Qi and Yin, according to TCM theory. The Yiqi Fumai freeze-dry powder (YFP) was here prepared based on the TCM formula of pulse-activating powder using modern production technology refined for formulation of modern Chinese medicine. The pulse-activating powder formula was taken from a comprehensive TCM book called Medicine Origin. It is one of the more famous TCM formulas for pulse activation. It consists of three active ingredients: ginseng, Mai Dong, and Chinese magnoliavine fruit. YFP has a beneficial impact on the replenishment of Qi and pulse activation. It also well nourishes Yin and promotes glandular secretion in TCM theory. Integrative Medicine may bring good results for those patients with Qi and Yin deficiency. However, there is still a lack of reliable clinical studies to prove the efficacy of this treatment. In this study, the Seattle Angina Scale (SAQ), ECG, stress echocardiography (stress echocardiography, SE) and amino-terminal pro-brain natriuretic peptide precursor (NT-proBNP) were used to assess the efficiency of YFP which was based on pulse-activating powder.

\section{Materials and Methods}

\subsection{Ethics}

This study was approved by hospital of Qingdao Municipal Hospital and Linyi People's Hospital Committee, and conformed to the guidelines of the National Institutes of Health for the care.

\subsection{Study Design}

100 patients diagnosed with unstable angina [1] (unstable angina pectoris, UAP) were selected randomly from 2011 to 2012 in both hospitals. Inclusion criteria of UAP cases: >18 years old; diagnosed with coronary heart disease (previous myocardial infarction, or revascularization history, at least one major vascular diameter stenosis $>50 \%$ by coronary angiography); or patients with the positive results of stress electrocardiogram test (male), radionuclide or stress echocardiography test; episode of angina; angina (seizure frequency, extent and level of incentives induced angina) has been stable for at least one at entrance; the Canadian Cardiovascular Society angina class I - IV grade. Exclusion criteria of UAP: patients developed acute myocardial infarction in less than three months; postoperative of revascularization (PCI or CABG) in less than three months, so 78 male patients and 22 female patients met these criteria. They were in line with the diagnostic criteria of ischemic diastolic heart failure (IDHF) [2]. Exclusion criteria of IDHF: patients with heart failure caused by non-coronary artery disease; heart failure symptoms aggravated due to digitalis poisoning.

All following patients were excluded: patients with severe hepatic or renal dysfunction (serum ALT levels $>3$ times the upper limit of the normal value, serum creatinine level $\geq 442 \mu \mathrm{mol} / \mathrm{L}$ ); uncontrolled hypertension (systolic blood pressure $\geq 180 \mathrm{mmHg}$, diastolic blood pressure $\geq 100 \mathrm{mmHg}$ ); Diabetic with glycemic level not controlled satisfactorily; previous history of allergy to ginseng, Ophiopogon japonicus, Schisandra chinensis; pregnant or lactating women.

All subjects signed the informed consent form. Grouping diagrammatic drawing was shown in Figure 1. 52 patients with TCM syndrome of qi-yin deficiency were accessed into Chinese and Western combination therapy group (chinese and western medicine treatment group, CWMTG) with 32 male and 20 female, and their ages were from 62 to 74 (average years were 69.5). 48 patients have no TCM syndrome of qi-yin deficiency including 8 females and 40 males with age from 57 to 70 (average years were $65.0 \pm 9.0$ ) were treated with standard western medicine (western medicine treatment group, WMTG).

UAP: unstable angina pectoris; TCM: raditional Chinese medicine; MTG: chinese and western medicine 


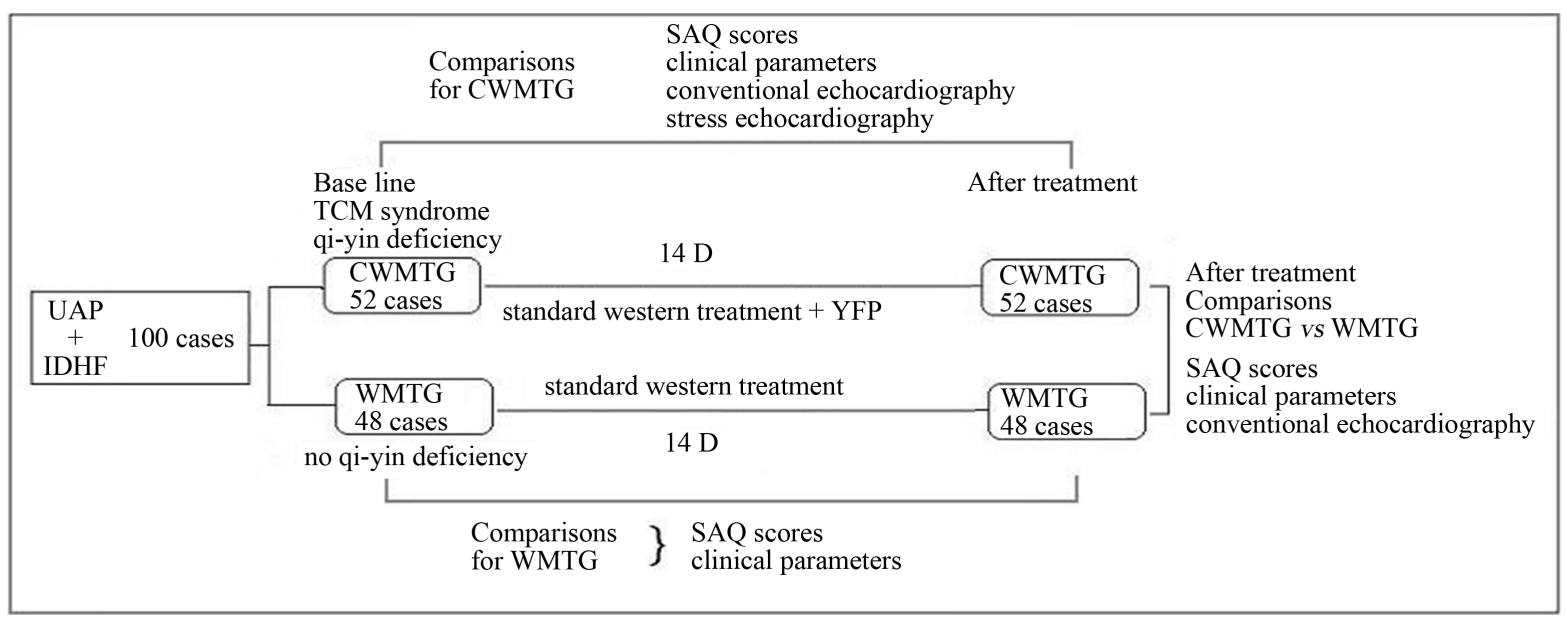

Figure 1. Study design and grouping.

treatment group; WMTG: western medicine treatment group; IDHF: ischemic diastolic heart failure; SAQ: seattle angina questionnaire.

\subsection{Treatment}

The standard western treatment includes the principles of conventional treatment of IDHF with diuretics, $\beta$-blockers, angiotensin-converting enzyme inhibitors (ACEI), angiotensin II receptor blockers (ARB); as well as standard ASC drugs such as aspirin, clopidogrel, isosorbide dinitrate and stantins. Patients in WMTG were given standard western treatment. On the basis of western medicine treatment, patients in CWMTG were given Yiqi Fumai dry powder (Tianjin Tasly Pharmaceutical Co., Ltd. Specification: $0.65 \mathrm{~g} /$ bottle. Approval Number: Z20060463). Dosage and Administration: $5.2 \mathrm{~g}$ each time, namely 8 bottles at a time, one time per day. The drugs were dissolved in $250-500 \mathrm{ml} 5 \%$ glucose injection or saline intravenous injection and were injected at the rate of about 40 drops per minute for 14 days).

\subsection{Seattle Angina Questionnaire (SAQ) Evaluation}

With the necessary explanations of SAQ by the doctor before and after treatment, patients independently completed the questionnaire. Seattle Angina scale evaluate the efficiency from following five aspects: physical limitation level (PL, Question 1), angina steady state (AS, Question 2), angina frequency (AF, Questions 3 - 4), treatment satisfaction (TS, Questions 5 - 8), disease perception (DP, Questions 9 - 11), one by one score, the total score is 100 points, the higher the score, the better the patient's quality of life and organism function [3].

\subsection{ECG Evaluation}

After treatment, disappearance of ischemic manifestation indicated in resting ECG or negative treadmill exercise test were considered markedly effective; that ST segment rebounded $0.05 \mathrm{mV}$, inverted T wave become shallow more than $50 \%$ or the inverted, low T wave become upright was effective; ST - T no change was invalid; ST segment descend, or $\mathrm{T}$ wave inversion deepens was aggravating.

\subsection{Stress Echocardiography}

Used Philips IE 33 ultrasonic diagnostic apparatus, X3-1 probe, 1-3MHz frequency, we recorded the left ventricular diastolic diameter (LVDd), interventricular sepal thickness at the end of diastolic (IVSd), left ventricular posterior wall thickness (LVPWd), left atria diameter (LAD). We measured the LVEF, the left ventricular output $(\mathrm{CO})$ and cardiac index (CI) by biplane Simpson on the parasternal long-axis under the resting standard state. Double-blind method was applied. All data measured repeatedly three times. The mean value was reserved. Patients that underwent stress echocardiography were asked to do treadmill exercise until their heart rates were stable at 110/min. After $1 \mathrm{~min}$, the test was terminated, and the indices were recorded at the same site. Indicators 
of cardiac Doppler echocardiography included cardiac systolic function LVEDV, LVESV, SV, CO, CI, EF, diastolic function E/A etc. All patients completed the test with satisfying results.

\subsection{NT-proBNP}

N-terminal pro brain natriuretic peptide (NT-proBNP) was detected by euzymelinked immunosorbent assay (biomelieux SA kit).

\subsection{Statistical Analysis}

SPSS11.5 statistical software was applied. All quantitative data were reported as mean \pm S.D. $(\bar{x} \pm s)$. Indicators between before and after treatment were compared using paired t-test, non-continuous variables between groups were compared using t-test. The continuous variables and rates were compared using $\mathrm{X}^{2}$ test. Non-parametric continuous variables were represented as the median (minimum - maximum). Paired Wilcoxon test was applied for comparisons before and after treatment; correlation between variables were analyzed using Pearson correlation analysis and independent factors were analyzed using multiple regression. $\mathrm{P}<0.05$ was as statistically significant.

\section{Results}

\subsection{SAQ Scores}

There were no significant differences of clinical general characteristics between two groups. Life quality of patients before and after treatment: scores of PL, AS, AF, TS, and DP are shown in Table 1. The results showed that the differences of PL, TS, DP between before and after treatment by YFP were statistically significant $(\mathrm{P}<$ 0.05), indicating that combination of YFP can improve physical limitation extent in patients with unstable angina, and can improve patients' satisfaction and their perception of the disease, making the patient's conditions to be stabilizing. The rate of nitrates use, ECG indicators, SAQ and symptoms were significant different before and after treatment $(\mathrm{P}=0.05)$. Patients' satisfaction and disease perception in CWMTG were significantly decreased $(\mathrm{P}=0.05)$ and the proportion of ineffectiveness in $\mathrm{CWMTG}$ was significantly decreased $(\mathrm{P}=0.02)$ (Figure 2 and Table 1$)$.
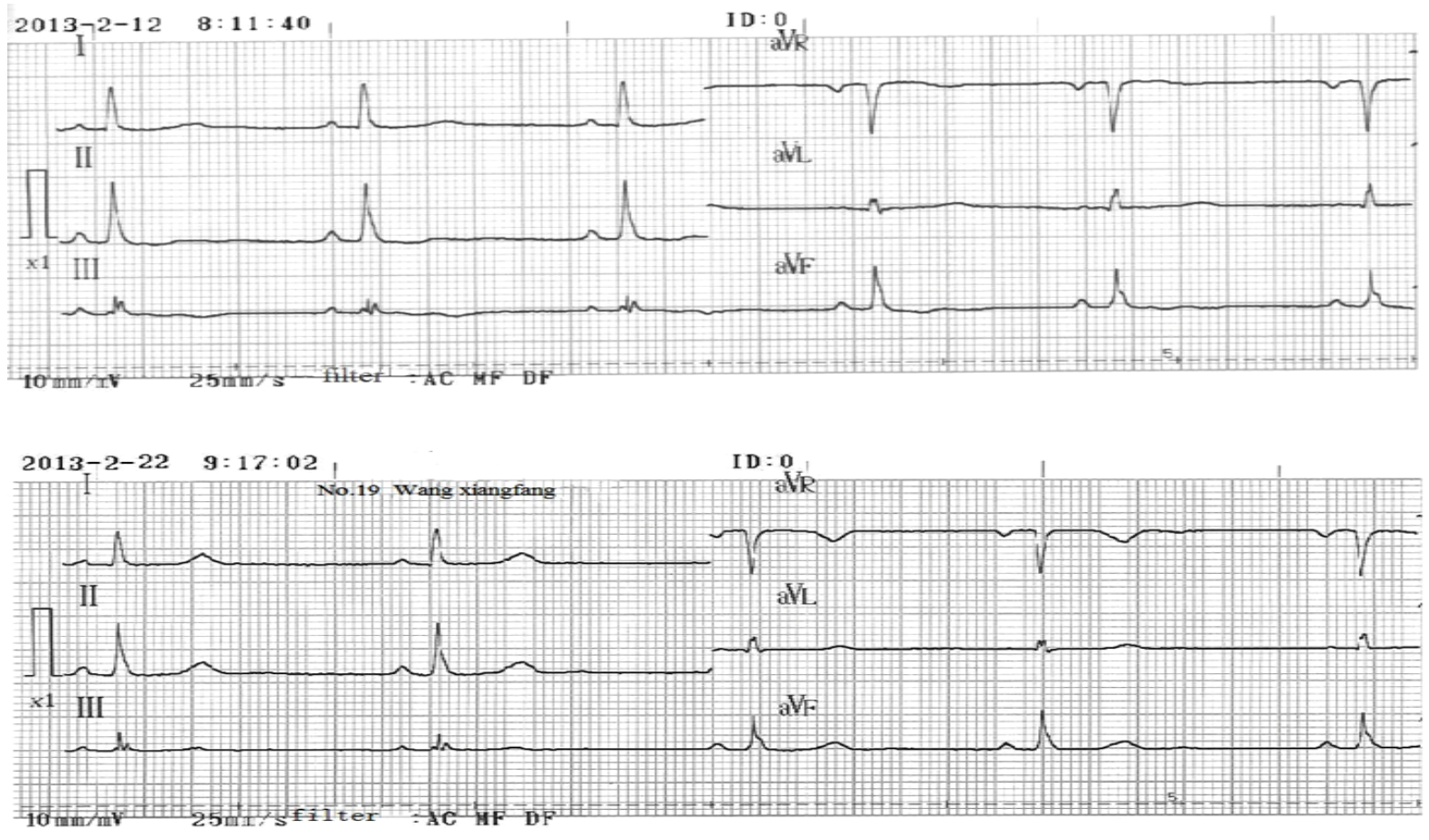

Figure 2. Changes of ECG of patients with IDHF before and after treatment. 
Table 1. Seattle angina questionnaire (SAQ) scores of two groups before and after treatment.

\begin{tabular}{|ccccccc}
\hline \multicolumn{2}{c}{ CWMTG $(\mathrm{n}=52)$} & & \multicolumn{2}{c}{ WMTG $(\mathrm{n}=48)$} & $\mathrm{P}^{*}$ \\
\hline Before treatment & After treatment & $\mathrm{P}$ & Before treatment & After treatment & P \\
\hline
\end{tabular}

General clinical characteristics

$\begin{array}{cc}\text { Age (years) } & 65.2 \pm 9.7 \\ \text { Male (\%) } & 76.4 \\ \text { Diabetes (\%) } & 28.2 \\ \text { Hypertension (\%) } & 39.5 \\ \text { Hyperlipidemia (\%) } & 60.5\end{array}$

Symptoms efficacy

$\begin{array}{cc}\text { Markedly } & 59.2 \\ \text { Effective } & 24.4 \\ \text { Ineffectiveness } & 16.4\end{array}$

\section{Treatment}

Anti-platelet (\%)
$\beta$-blockers (\%)
statins (\%)
ACEI/ARB (\%)
Nitrates (\%)

SAQ

\begin{tabular}{|c|c|c|c|c|c|c|c|}
\hline PL & $64 \pm 22$ & $54 \pm 25$ & 0.02 & $65 \pm 23$ & $60 \pm 24$ & 0.05 & 0.04 \\
\hline AS & $40 \pm 34$ & $37 \pm 31$ & 0.05 & $39 \pm 30$ & $33 \pm 20$ & 0.05 & 0.55 \\
\hline $\mathrm{AF}$ & $66 \pm 27$ & $59 \pm 27$ & 0.03 & $67 \pm 26$ & $61 \pm 25$ & 0.03 & 0.75 \\
\hline $\mathrm{TS}$ & $89 \pm 18$ & $96 \pm 16$ & 0.001 & $88 \pm 16$ & $90 \pm 17$ & 0.004 & 0.05 \\
\hline DP & $42 \pm 22$ & $48 \pm 23$ & 0.05 & $44 \pm 24$ & $49 \pm 22$ & 0.05 & 0.05 \\
\hline
\end{tabular}

P: Comparisons of each group before and after treatment; $P^{*}$ : Comparisons of the two groups after treatment.

\subsection{Comparisons of Clinical Baseline Data of Patients with IDHF before and after Treatment}

Clinical baseline characteristics of patients in CWMTG before and after treatment are shown in Table 2. After treatment, plasma NT-proBNP levels and the rate of $\beta$-blocker's use were decreased, the exercise tolerance increased. The differences were statistically significant. The rate of diuretics and lipid-lowering drugs use reduced without statistical significance. Plasma NT-proBNP levels of patients in CWMTG decreased significantly after treatment $(70.5(62.0$ - 79.0) ng/L vs $74.0(65.0$ - 84.0) ng/L, P < 0.05). After treatment, plasma NT-proBNP levels of patients in WMTG were not significantly changed (Table 2).

\subsection{Changes of Echocardiography of Patients with IDHF before and after Treatment}

Comparisons of echocardiography parameters of 52 patients with DHF before and after combination therapy were shown in Table 3. The results showed that after treatment, SE parameters of LVEF, E/A were significantly increase; E/e' decreased. The differences were statistically significant. However echocardiography parameters showed no significant differences after treatment (Figure 3 and Table 3). 


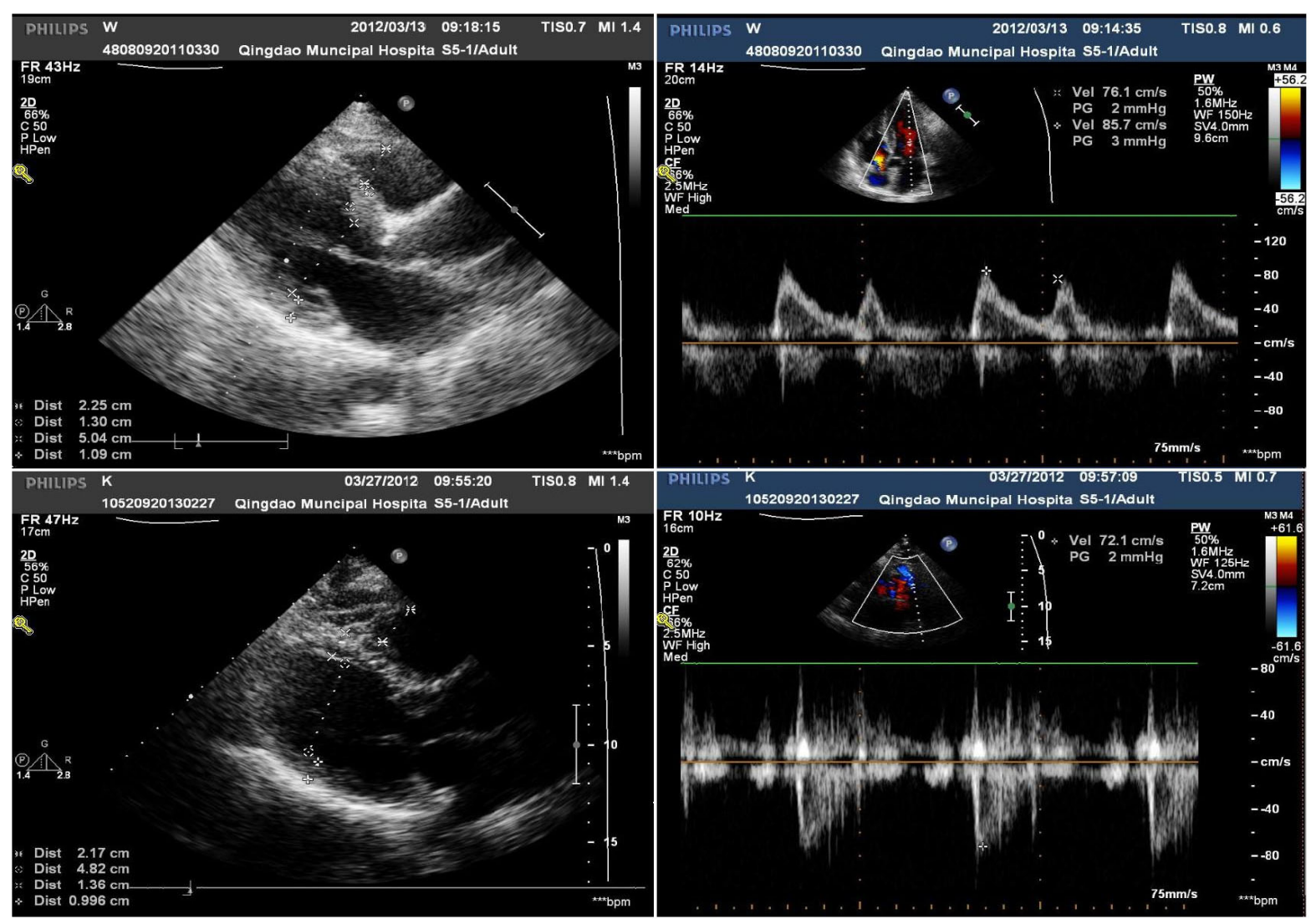

Figure 3. Changes of echocardiography of patients with IDHF before and after treatment.

Table 2. comparisons of clinical parameters of 52 patients with ischemic diastolic heart failure patients before and after treatment.

\begin{tabular}{|c|c|c|c|}
\hline Clinical parameters & Before treatment & After treatment & $\mathbf{P}$ \\
\hline \multicolumn{4}{|l|}{ Demographic characteristics } \\
\hline Age & 69. $(62.0-74.0)$ & - & - \\
\hline Male (\%) & $32(61.5 \%)$ & - & - \\
\hline Hypertension (\%) & $33(63.5 \%)$ & - & - \\
\hline Diabetes (\%) & $12(23.1 \%)$ & - & - \\
\hline Hyperlipidemia (\%) & $35(67.3 \%)$ & - & - \\
\hline Fat $(\%)$ & $19(36.5 \%)$ & - & - \\
\hline $\operatorname{BMI}\left(\mathrm{kg} / \mathrm{m}^{2}\right)$ & $29.5(26.0-33.0)$ & - & - \\
\hline \multicolumn{4}{|l|}{ Treatment } \\
\hline Diuretics (\%) & $27(52.6 \%)$ & $20(38.5 \%)$ & 0.737 \\
\hline$\beta$-blockers $(\%)$ & $40(76.3 \%)$ & $40(54.7 \%)$ & 0.032 \\
\hline Lipid-lowering drugs (\%) & $33(63.2 \%)$ & $27(52.8 \%)$ & 0.325 \\
\hline ACEI/ARB (\%) & $36(68.4 \%)$ & $35(67.9 \%)$ & 0.960 \\
\hline NT-proBNP (ng/L) & $250.7(167.5-544.0)$ & $94.3(67.0-151.5)$ & $<0.001$ \\
\hline \multicolumn{4}{|l|}{ Hemodynamic } \\
\hline Heart rate (bpm) & $70.5(62.0-79.0)$ & $74.0(65.0-84.0)$ & 0.045 \\
\hline Systolic blood pressure (mmHg) & $130.0(120.0-150.0)$ & $130.0(120.0-140.0)$ & 0.042 \\
\hline Diastolic blood pressure $(\mathrm{mmHg})$ & $82.5(80.0-95.0)$ & $80.0(80.0-90.0)$ & 0.158 \\
\hline \multicolumn{4}{|l|}{ Muscle testing } \\
\hline Exercise tolerance (s) & $387.0(305.0-482.0)$ & $463.0(382.0-527.0)$ & $<0.001$ \\
\hline
\end{tabular}

P: Comparisons of before and after treatment. 
Table 3. Comparisons of conventional and stress echocardiography index of 52 patients with ischemic diastolic heart failure patients before and after treatment.

\begin{tabular}{|c|c|c|c|c|}
\hline The indexes & $\begin{array}{c}\text { Before treatment } \\
\text { Echo }\end{array}$ & $\begin{array}{c}\text { After treatment } \\
\text { Echo }\end{array}$ & $\begin{array}{c}\text { After treatment } \\
\text { SE }\end{array}$ & $\mathrm{P}$ \\
\hline \multicolumn{5}{|c|}{$\begin{array}{l}\text { Two-dimensional } \\
\text { echocardiography }\end{array}$} \\
\hline $\operatorname{EDVI}\left(\mathrm{mL} / \mathrm{m}^{2}\right)$ & $45.0(39.0-54.0)$ & $45.0(39.9-50.0)$ & $45.0(40.5-49.5)$ & 0.717 \\
\hline $\operatorname{ESVI}\left(\mathrm{mL} / \mathrm{m}^{2}\right)$ & $16.0(12.0-21.0)$ & $15.2(12.0-18.9)$ & $14.0(11.0-16.5)$ & 0.167 \\
\hline LVEF (\%) & $54.0(50.0-60.0) \mathrm{a}$ & $58.0(70.0-66.0) \mathrm{a}$ & $69.5(66.5-73.0) b$ & 0.000 \\
\hline $\operatorname{LVMI}\left(\mathrm{g} / \mathrm{m}^{2}\right)$ & $102.5(88.0-123.0) \mathrm{a}$ & $101.4(83.0-110.6) \mathrm{a}$ & $89.0(72.0-93.5) b$ & $<0.001$ \\
\hline $\operatorname{LAVI}\left(\mathrm{mL} / \mathrm{m}^{2}\right)$ & $36.0(29.0-41.0) \mathrm{a}$ & $34.0(28.0-37.9) \mathrm{a}$ & $28.0(24.0-32.0) b$ & 0.001 \\
\hline LVDd & $54.7(50.2-55.0)$ & $53.5(49.7-54.1)$ & $51.0(46.2-53.0)$ & 0.03 \\
\hline \multicolumn{5}{|l|}{ Left ventricular } \\
\hline \multicolumn{5}{|l|}{ Doppler } \\
\hline $\mathrm{E}(\mathrm{cm} / \mathrm{s})$ & $77.5(67.0-94.0)$ & $76.0(65.0-96.3)$ & $70.0(61.5-80.0)$ & 0.179 \\
\hline E-exe $(\mathrm{cm} / \mathrm{s})$ & $107.5(94.0-126.0) \mathrm{a}$ & $105.2(92.3-120.2) \mathrm{a}$ & $96.0(83.5-102.5) b$ & 0.005 \\
\hline $\mathrm{A}(\mathrm{cm} / \mathrm{s})$ & $92.0(79.0-105.0) \mathrm{a}$ & $90.1(72.2-101.4) \mathrm{a}$ & $72.0(65.0-79.0) b$ & $<0.001$ \\
\hline A-exe $(\mathrm{cm} / \mathrm{s})$ & $103.0(92.0-121.0) \mathrm{a}$ & $101.3(90.8-118.7) \mathrm{a}$ & $97.5(87.0-109.5) a, b$ & 0.041 \\
\hline $\mathrm{E} / \mathrm{A}$ & $0.8(0.7-1.0) \mathrm{a}$ & $0.8(0.5-1.1) \mathrm{a}$ & $1.0(0.9-1.1) b$ & 0.016 \\
\hline E-exe/A-exe & $1.0(0.9-1.2)$ & $1.0(0.8-1.2)$ & $0.9(0.8-1.1)$ & 0.826 \\
\hline $\mathrm{DT}(\mathrm{ms})$ & $189.0(167.0-208.0) \mathrm{a}$ & $181.2(161.2-203.0) \mathrm{a}$ & $159.5(149.5-189.0) b$ & 0.017 \\
\hline Ard-Ad (ms) & $-10.0(-30.5-(-1.5))$ & $-11.3(-30.0-(-2.2))$ & $-10.5(-17.0-(-5.0))$ & 0.640 \\
\hline $\mathrm{e}^{\prime}(\mathrm{cm} / \mathrm{s})$ & $7.1(6.3-8.4) \mathrm{a}$ & $7.9(6.9-9.2) \mathrm{a}$ & $9.4(8.8-10.5) b$ & $<0.001$ \\
\hline$e^{\prime}-\operatorname{exe}(\mathrm{cm} / \mathrm{s})$ & $9.3(8.1-10.8) \mathrm{a}$ & $9.8(9.5-12.9) \mathrm{a}$ & $12.9(12.0-14.3) b$ & $<0.001$ \\
\hline $\mathrm{a}^{\prime}(\mathrm{cm} / \mathrm{s})$ & $9.8(8.4-11.0) \mathrm{a}$ & $10.2(8.9-11.7) \mathrm{a}$ & $11.2(9.6-12.6) b$ & 0.012 \\
\hline $\mathrm{a}^{\prime}-\operatorname{exe}(\mathrm{cm} / \mathrm{s})$ & $11.1(9.2-13.3) \mathrm{a}$ & $12.5(10.9-14.2) \mathrm{a}$ & $14.4(13.0-16.5) \mathrm{c}$ & $<0.001$ \\
\hline $\mathrm{s}^{\prime}(\mathrm{cm} / \mathrm{s})$ & $7.6(7.0-8.2) \mathrm{a}$ & $7.9(7.8-8.6) \mathrm{a}$ & $8.8(8.0-9.3) b$ & $<0.001$ \\
\hline $\mathrm{s}^{\prime}-\operatorname{exe}(\mathrm{cm} / \mathrm{s})$ & $9.5(8.8-10.3) \mathrm{a}$ & $10.5(8.9-13.2) \mathrm{a}$ & $13.9(12.5-15.5) \mathrm{c}$ & $<0.001$ \\
\hline $\mathrm{E} / \mathrm{e}^{\prime}$ & $10.6(9.4-12.7) \mathrm{a}$ & $10.4(9.0-10.9) \mathrm{a}$ & $7.0(6.0-8.9) b$ & $<0.001$ \\
\hline E-exe/e'-exe & $11.0(9.9-12.5) \mathrm{a}$ & $10.0(8.9-11.2)$ & $7.2(6.5-7.7) b$ & $<0.001$ \\
\hline
\end{tabular}

\subsection{Main Echocardiography Parameters of CWMTG Patients and NT-proBNP Correlation Analysis}

After the combination treatment, NT-proBNP level had negative correlation with LVEF, E/A, r $=-0.432$, -0.643 , both $\mathrm{P}<0.01$. After treatment, multiple stepwise regression analysis of NT-proBNP and cardiac function indexes showed that the left ventricular end-diastolic diameter (LVDd), left ventricular ejection fraction (LVEF), early diastolic mitral flow velocity (E), DT, left ventricular isovolumic relaxation time (IVRT), E/A were independent relevant factors of NT-proBNP (Table 4).

\section{Discussions}

In TCM, heart failure is divided into the categories of edema, palpitation, gasp syndrome, phlegm and fluid retention, and blood stasis. Therapy combining Chinese and Western medicine may have pronounced efficacy in the treatment of patients with unstable angina who have been diagnosed with Yin and Qi deficiencies by TCM differentiation. However, there are a few of studies confirming the efficacy of this combination therapy. Studies focusing on objective indicators of improved myocardial function, such as the evaluation index of DHF, regardless of self-reported symptoms of the patients, would be especially useful. In the present work, the SAQ, ECG, stress echocardiography (SE), and the NT-proBNP were all used to assess the efficacy of YFP, which was prepared based on the TCM formula of pulse-activating powder. Assessment of SAQ suggested that treatment with CWMTG brought about significant improvements, including reduced number and severity of angina attacks, and improved the quality of life. In addition, ECG analysis indicated that CWMTG had a more pronounced curative effect than isosorbide denigrate treatment. The results of the SE demonstrated that all patients had symptoms of heart failure and that their LVEF was $>50 \%$ and the ration of early (E) and late (A) transmittal diastolic velocity 
Table 4. Multiple stepwise regression analysis of NT-proBNP and cardiac function indexes of 52 patients after treatment.

\begin{tabular}{|c|c|c|c|c|}
\hline Indexes & Non-standardized coefficients & Standardized coefficients & $\mathbf{t}$ & $\mathbf{P}$ \\
\hline \multicolumn{5}{|c|}{$\begin{array}{l}\text { Two-dimensional } \\
\text { echocardiography }\end{array}$} \\
\hline LVEF & $1.972^{\circ}$ & $0.147 \mathrm{~b}$ & 3.487 & 0.001 \\
\hline LVDd & 8.651 & 0.131 & 3.399 & 0.001 \\
\hline \multicolumn{5}{|c|}{ Left ventricular } \\
\hline \multicolumn{5}{|l|}{ Doppler } \\
\hline $\mathbf{E}$ & $6.150^{\circ}$ & $0.254 \mathrm{a}, \mathrm{b}$ & 6.260 & 0.000 \\
\hline $\mathrm{E} / \mathrm{A}$ & 0.104 & $0.097 \mathrm{~b}$ & 2.616 & 0.009 \\
\hline DT & 2.296 & 0.168 & 3.498 & 0.001 \\
\hline
\end{tabular}

a: compared with before treatment, $\mathrm{P}<0.05$. b: compared with conventional echocardiography, $\mathrm{P}<0.05$.

(E/A ratio) was $<1$, suggesting that the patients suffered from DHF. After treatment with CWMTG, the relative number of irregular inter-ventricular sepal (IVS) and LVPW movements was significantly went down. LVEF, $\mathrm{CO}$ and CI were significantly increased and the patients showed enhanced diastolic function (E/A ratio). All of these indicated that the LVMI was reduced. Systolic function and diastolic function were improved, and stress echocardiography is more sensitive than conventional echocardiography for evaluating the therapeutic effect of diastolic heart failure.The blood levels of NT-proBNP in the patients of the CWMTG were significantly lower than that in controls. The patients also showed improved exercise tolerance and some of them were able to reduce their doses of diuretics and lipid-lowering drugs.

Clinical symptoms of UAP are chest tightness and pain, chest pain involving the back, back pain involving the chest, shortness of breath, gasping, and restlessness. In severe cases, the disease may lead to death within hours of the initial onset of symptoms. The etiological factors are mainly invasion of pathogenic cold, eating disorders, mental stress and extreme changes in emotional state, disorders of the internal organs caused by fatigue, and old age. The location of the disease is mainly the heart and pathogenesis is heart vessel blockage stasis. In the TCM symptom classification system, patients with the mild form of the disease have hypo function of Yang-Qi in the chest, excessive cold syndrome, and blockage of Qi. Patients with severe form of the disease have accumulation of blood-phlegm, congestion in the chest, and blockage of Qi. Delays in treatment may cause damage to Yin and Yang and deficiencies in Qi and Yin, according to TCM theory. The active ingredients of YFP include Radix Ginseng Rubra (Panax ginseng or red ginseng), Radix Ophiopogonis (Mai Dong), and Fructus Schisandrae (Chinese magnoliavine fruit). Red ginseng reinforces vital energy of body, improves lung function, and promotes glandular secretion. It also provides fixation and hidroschesis. It also provides the principle curative action (sovereign drug). The effects of Mai Dong can nourish Yin and relieve dryness of the body. Combination of this herb with red ginseng may notify both Qi and Yin. Mai Dong is also used as minister drug. Chinese magnoliavine fruit can astringe Yin to stop sweating. Combinations of this herb with red ginseng and Mai Dong may consolidate Qi and glandular secretion to prevent leakage and promote recovery from wastage of Qi and Yin. For this reason, it is also termed an assistant drug, an adjuvant drugs that increases the efficacy or potency of sovereign and minister drugs in TCM. These three herbs are combined: to resolve Qi deficiency using red ginseng as a basis; to prevent Qi leakage using Chinese magnoliavine fruit as a standard; and to replenish Yin using Mai Dong. This eventually allows primordial Qi to be ventilated in the lungs, Yin to be restored, and the pulse to be normalized.

Modern pharmacological research has confirmed that the active ingredients of ginseng extracts (panaxosides or ginsenosides) promote DNA and RNA syntheses, increasing hypoxia tolerance, enhancing myocardial contractility, increasing plasma levels of cyclic adenosine monomphsopate (cAMP) and the synthesis of prostaglandin $\mathrm{I}_{2}$, or $\mathrm{PGI}_{2}$, thus inhibiting platelet aggregation and improving myocardial cell metabolism [4]. Mai Dong stabilizes myocardial cell membranes with a positive inotropic effect and eliminates free radicals [5]. Chinese magnoliavine fruit strengthens myocardial contractions, improves microcirculation and inhibits lipid peroxidatiuon. Combinations of these three herbs may enhance myocardial contractility, improve left ventricular function and tolerance of myocardial hypoxia, and protect the injured myocardial ultrastructure [6]. YFP injection can tonify Qi and activate blood circulation; reduce plasma viscosity: promote fibrinolysis; inhibit thrombosis; im- 
prove abnormal hemorheology, thereby relieving angina and improving self-reported symptoms [7].

Inhibition of $\mathrm{Na}^{+}-\mathrm{K}^{+}$-ATPase activity affects $\mathrm{Na}^{+}-\mathrm{K}^{+}$and $\mathrm{Na}^{+}-\mathrm{Ca}^{2+}$ exchange, thereby increasing $\mathrm{Ca}^{2+}$ influx and $\mathrm{Ca}^{2+}$ concentration. This promotes the interaction of $\mathrm{Ca}^{2+}$ with cardiac troponin, resulting in increases in myocardial contractility and cardiac output [8]. Clinical studies published by Zhong et al. demonstrated that pulse-activating injection enhances the capacity of myocardial contraction in patients with ischemic heart disease [9]-[11]. These patients also showed improvement in myocardial compliance and coordination, increased perfusion pressure, and a higher myocardial survival rate and ejection fraction. In the present study, patients' LVEF values were significantly increased and their plasma levels of NT-proBNP were significantly reduced after YFP treatment, suggesting that YFP treatment efficiently improves cardiac function. In addition, these findings are consistent with those of other studies performed using in vivo models. In these studies, pulse-activating injection enhanced patients' myocardial contractility and vasodilation but did not increase the heart rate (all regimens either reduced the heart rate or left it unchanged) [12] [13].

It is not subject to the insufficiencies of more commonly used quality of life scales, which do not sufficiently address the specific conditions or psychology of CAD patients, lack internal consistency in some areas, and have poor response to treatment intervention [14]. Repeated assessments involving the SAQ scale has demonstrated its strong reproducibility. In TCM, efficacy is assessed mostly via clinicians' observations of improvements and patient recovery after the examination through TCM's four diagnostic methods of TCM, observation, auscultation and olfaction, interrogation, and assessments of pulse and palpation. However, these assessments do not consider the quality of life of the patients (e.g., patient's comfort, subjective feelings, and degree of life satisfaction). Quality of life does not only represent and integrate personal values with judgment of health status and life satisfaction but also reveals the patient's expectations with respect to the efficacy of the treatment and improvements in function [15].

The SAQ shows that YFP effectively reduces the frequency of clinical attacks of UAP and facilitates significant improvements in UAP self-reported symptoms, quality of life, and patient satisfaction. However, most of the clinical research to date has only focused on adjuvant TCM therapy on the basis of conventional Western medicine. The lack of evidence-based medicine that can be used to assess the clinical efficacy of an individual TCM regimen or TCM regimen used in place of standard UAP drug therapy complicates the assessment of specificity and definite efficacy of TCM treatment. In addition, the standard treatment for UAP has a standardized duration of follow-up after drug administration. However, there are no published reports addressing the long-term use of TCM and duration of TCM administration in this disease. The present work study confirmed that YFP was associated with improvements in patients' self-reported symptoms of UAP and in patients' satisfaction after treatment. It showed efficacy superior to that of a treatment with Western medicine, so it may be possible to use YFP to compensate for some of the insufficiencies of conventional Western medicine.

During myocardial ischemia, abnormalities in cardiac diastolic function occur prior to systolic dysfunction. Left ventricular diastole includes isovolumetric relaxation (early diastole) and ventricular filling phases. The isovolumetric relaxation phase and rapid filling phase involve active energy-consuming processes; but in the slow filling phase and atria systole phase, the ventricle was passively diastole, which was dependent on the stiffness of the left ventricular myocardial muscle. Conventional echocardiography is not sensitive enough to facilitate prognosis or assessment of the efficacy of treatment. This can lead to misdiagnosis and missed diagnoses. In this way, conventional echocardiography has significant limitations. Stress echocardiography (SE) is a new technique that combines a treadmill exercise stress test and an echocardiography analysis. It is a functional test that involves allowing the patient to exercise to increase myocardial oxygen consumption, which induces pronounced homodynamic changes and increases the detection rate of coronary heart disease while maintaining high sensitivity, specificity, and accuracy for the detection of myocardial ischemia. Because of its noninvasiveness, convenience, repeatability, and ability to determine the homodynamic changes in patients, SE can be used to guide treatment and perform prognosis. In this way, it is one of the more reliable and objective tests to have achieved widespread use in clinical settings in recent years. Although all of the patients in this study had DHF with normal systolic function, SE still demonstrated the changes in myocardial systolic function after YFP treatment. LVEF and LVMI changed significantly after YFP treatment, suggesting enhancement of systolic function and further illustrating that SE allows for a comprehensive evaluation of the therapeutic effect. However, evaluation with left ventricular Doppler flow spectrum was affected by heart rate, preload (left atria pressure) and after load (aortic pressure). Exercise increases heart rate, which may affect the results of left ventricular Doppler flow spectrum and may merit further analysis. In this way, SE also has some limitations. The key to 
improve the sensitivity of SE is correct detection of load-induced segmental wall motion abnormalities (SWMAs). SWMAs occur only during myocardial ischemia. Sometimes, even under circumstances involving severe coronary artery stenosis, SWMAs can remain undetected if myocardial ischemia is not induced by load. In addition, the limitations of traditional tissue Doppler imaging include inability to measure myocardial motions at different sites at the same time and an inability to detect myocardial velocity gradient. These issues should be addressed in future studies.

Modern medicine suggests that heart failure is not only related to homodynamic abnormalities but also associated with the activation of the neuroendocrine system. The NT-proBNP is a cardiac neurohormone. In the human body, it plays a role in diuresis, natriuresis, and vasodilatation. Declined cardiac output, increased tension of the ventricular wall, and overloaded pressure induce the secretion of NT-proBNP, and levels of NT-proBNP can be indicative of the overall situation of circulatory congestion. There is a positive correlation between the NT-proBNP value and the severity of heart failure, indicating that compensatory pathophysiological changes have taken place. The NT-proBNP value is a marker of the state of restoration of the balance of circulation in human body, and it is negatively correlated with the quality of cardiac function [16]. This study suggested that the NT-proBNP is negatively correlated with LVEF and E/A $(r=-0.432$ and -0.643$)$. Left ventricular diastolic dysfunction (LVDd), LVEF, E, diastolic (D wave) flow velocity, isovolumic relax-action time (IVRT), and E/A ratio were found to be independent factors associated with NT-proBNP, suggesting that the increase of left ventricular end-systolic pressure (LVESP) and the diastolic dysfunction may result in increased production of NT-proBNP. The changes in NT-proBNP levels may represent the systolic and diastolic functions of the heart. These results are consistent with the SE evaluation of the severity of heart failure. However, NT-proBNP can distinguish neither the cause of heart failure nor the type of heart failure; whereas SE can compensate this inadequacy, that is to say, SE can distinguish the cause and the type of heart failure. This study examined the comprehensive evaluation of cardiac function using patients' SE and plasma NT-proBNP levels. These methods were found to facilitate diagnosis of chronic heart failure and evaluation of its severity in a more reasonable and accurate manner than other methods. These methods were also used to diagnose the underlying heart conditions of the patients, suggesting that the evaluation in these patients using SE and plasma NT-proBNP index was reliable and sensitive.

This study confirmed that YFP treatment improves self-reported symptoms, increases the degree of satisfaction, and makes up for the drawbacks of standard Western medical regimen. It was here confirmed that SAQ is sensitive and suitable for evaluation of the curative effects of TCM, as been shown in a previous study [17].

Stress echocardiography provides some parameters for evaluation of the changes in IDHF and can serve as a quantitative indicator of the clinical efficacy of YFP treatment. The present study confirmed that YFP treatment is reliable and can improve cardiac function and the exercise tolerance in patients. In this study, we didn't discuss whether YFP is effective to all patients with ischemic diastolic heart failure or just effective to ischemic diastolic heart failure patients with with TCM syndrome of qi-yin deficiency. Besides, the sample cases were too little, we should increase the cases to make the results reliable.Improvements in the SE index, levels of NT-proBNP and clinical symptoms in the patients remained consistent throughout this study, suggesting that the combination of these methods may be helpful for research into integrative therapy involving TCM and Western medicine.

\section{References}

[1] Cardiovascular Medicine Branch of Chinese Medical Doctor Association, Evidence-Based Medicine Branch of Chinese Medical Doctor Association Commition (2007) Guidelines and Consensus on Prevention and Treatment of Cardiovascular Diseases 2007[M]. People's Medical Publishing House, Beijing, 37-39.

[2] Young, M.N., Shoemaker, M.B., Kurtz, E.G., et al. (2012) Heart Failure with Preserved Left Ventricuiar Function: Diagnosticand Therapeutic Challenges in Patients with Diastolic Heart Failure. The American Journal of the Medical Sciences.

[3] Spertus, J.A., Winders, J.A., Dewhurst, T.A., et al. (1995) Development and Alidation of the Seattle Angina Questionnaire: Journal of the American College of Cardiology, 25, 333-341. http://dx.doi.org/10.1016/0735-1097(94)00397-9

[4] Yang, W.M. and Zhou, Y.X. (1997) Clinical Observation on Treatment of Chronic Congestive Heart Failure with Shenmai Injection: 62 Cases. Practical Journal of Integrated Traditional Chinese and Western Medicine, 10, 1446.

[5] Wang, J.H. (1994) Handbook of Commonly Used Traditional Chinese Medicine [M]. The Golden Shield Press, Beijing, 112. 
[6] Xu, L.H. (2009) Therapeutic Effect of Yiqi Fumai in Patients with Chronic Congestive Heart Faliure. Clinical Medicine, 22, 2418-2419.

[7] Yuan, C.L. and Du, S.L. (2012) Efficacy of Yi Qi Fu Mai Injection on Heart Failure Complicated with Angina Pectoris in Patients with Coronary Heart Disease. Chinese Journal of New Drugs, 1774-1777.

[8] Mao, J.Y., Zhang, B.L. and Wang X.L. (2006) Progress in Mechanism of Shenmai Injection in the Treatment of Heart Failure. Chinese Traditional Patent Medicine, 28, 1801-1803.

[9] Dong, Q.Z., Chen, K.Y. and Tu, X.H. (1984) Hemodynamic Effects of Shengmai Injection in the Treatment of Acute Myocardial Infarction. Chinese Journal of Cardiology, 12, 5-6.

[10] Zhong, Y.S. (1998) Effect of Shengmai Injection on Left Ventricular Volume and Function of Patients with Heart Failure after Acute Myocardial Infarction. Journal of Wuhan Postgraduate Medical College, 26, 1-2.

[11] Zhuang, A.L. and Guan, E.J. (1997) Effect of "Sheng Mai" Injection on Cardiac Function of Patients with Ischemic Heart Disease. Chinese Journal of Integrated Traditional and Western Medicine in Intensive and Critical Care, 4, 310-311.

[12] Department of Chest Emergency Coordination Group of the State Administration of Traditional Chinese Medicine Medical Administration. (1995) Clinical and Experimental Studies of Shenmal Injection for Treatment of 219 Cases of Angina Pectoris of CHD. Journal of Emergency in Traditional Chinese Medicine, 4, 152-155.

[13] Meng, Q.Y. (2000) Preliminary Study on Rapid Injection of Shengmai Injection in the Treatment of Hypertensive Emergencies. Hebei Medicine, 6, 133-134.

[14] Xing, W.H. and Chen, X.M. (2004) Feasibility of SF-8 Short Scale for Testing Health Related Quality of Life in Patients with Coronary Heart Disease. Foreign Medical Sciences (Section of cardiovascular), 5, 181-184.

[15] Hao, Y.T., Fang, J.Q., Li, C.X., et al. (1999) World Health Organization. WHOQOL and Its Chinese Version. Foreign Medical Sciences (Section of Social Medicine) 16, 118-122.

[16] Wang, L., Hu, Y.S., Wu, X., et al. (2010) Significances of NT-proBNP and hs-CRP in Heart Failure. National Medical Journal of China, 90, 1635-1636.

[17] Liu, T.X., Kong, S.P., Liao, Z.Y., et al. (1997) Assessment Study on Physical Function and the Quality of Life for CHD Patients with SAQ. Chinese Journal of Behavioral Medical Science, 6, 127-129.

\section{Abbreviation}

IHF: ischemic heart failure

YFP: Yiqi Fumai freeze-dry powder

TCM: traditional Chinese medicine

IDHF: ischemic diastolic heart failure

CWMTG: Chinese and western medicine treatment group

WMTG: western medicine treatment group

SAQ: Seattle Angina Questionnaire

AMI: acute myocardial infarction

DHF: diastolic heart failure

SE: stress echocardiography

UAP: unstable angina pectoris

ACS: acute coronary syndrome

PCI: percutaneous transluminal coronary intervention

CABG: Coronary artery bypass grafting

ACEI: angiotensin-converting enzyme inhibitors

ARB: angiotensin II receptor blockers

LVDd: left ventricular diastolic diameter

IVSd: interventricular sepal thickness at the end of diastolic

LVPWd: left ventricular posterior wall thickness

LAD: left atria diameter

LVEF: left ventricular ejection fraction

$\mathrm{CO}$ : left ventricular output 
CI: cardiac index

IVS: inter-ventricular sepal

SWMAs: segmental wall motion abnormalities

IVRT: isovolumic relax-action time

LVESP: left ventricular end-systolic pressure

LAVI: left atrial volume index

EDVI: left ventricular end-diastolic volume

ESVI: systolic volume index;

LVMI: left ventricular mass index

E: early diastolic two mitral flow velocity

A: late diastolic mitral flow velocity

E / e ': early diastolic mitral flow velocity and early diastolic mitral annular velocity ratio

DT: mitral E wave deceleration Time

Ard - Ad: aortic root diameter.

Echo: conventional echocardiography

SE: stress echocardiography

ECG: electrocardiogram

NT-proBNP: N-terminal pro brain natriuretic peptide 